\title{
Reversible DNA codes from skew cyclic codes over a ring of order 256
}

\section{Yasemin Cengellenmis, Nuh Aydin, Abdullah Dertli}

Abstract: We introduce skew cyclic codes over the finite ring $\mathbb{F}_{2}+u \mathbb{F}_{2}+v \mathbb{F}_{2}+w \mathbb{F}_{2}+u v \mathbb{F}_{2}+u w \mathbb{F}_{2}+v w \mathbb{F}_{2}+u v w \mathbb{F}_{2}$, where $u^{2}=0, v^{2}=v, w^{2}=w, u v=v u, u w=w u, v w=w v$ and use them to construct reversible DNA codes. The 4-mers are matched with the elements of this ring. The reversibility problem for DNA 4-bases is solved and some examples are provided.

2010 MSC: 94B05, 94B60

Keywords: DNA codes, Skew codes, Reversibility

\section{Introduction}

It is well known that DNA contains genetic program for the biological development of life and has two strands which are linked by Watson-Crick pairing so that every $A$ is linked with a $T$ and every $C$ with a $G$, and vice versa, where $A, T, C, G$ are the four bases of a DNA sequence.

DNA computing started in 1994 when Adleman showed how to solve a computationally difficult problem (traveling salesman problem, a well-known NP-complete problem) by manipulations of DNA molecules in [2]. Later, more applications of DNA codes were discovered such as using DNA codes to break a cryptosystem known as DES [3, 5], and using DNA codewords as high density storage media [16]. Devising methods to design DNA codes for DNA computing has been a major topic of research since the beginning of the century. A block code is called a DNA code if it satisfies the following constraints $[8,22]$.

1. the Hamming constraint for minimum distance,

Yasemin Cengellenmis; Department of Mathematics, Trakya University, Edirne, Turkey (email: ycengellenmis@gmail.com).

Nuh Aydin; Department of Mathematics and Statistic, Kenyon College, Gambier,OH, United States (email: aydinn@kenyon.edu).

Abdullah Dertli (Corresponding Author); Department of Mathematics, Ondokuz Mayıs University, Samsun, Turkey (email: abdullah.dertli@gmail.com). 
2. the reverse-complement constraint,

3. the reverse constraint, and

4. the fixed GC content.

In [8], all of these constraints are translated in terms of coding theory. This enabled researchers to use the results from classical coding theory to design codes for DNA computation. The last decade witnessed an increased activity in this direction. Since DNA uses a four-letter alphabet, $\{A, G, T, C\}$, it is most natural to use a ring of size 4 in employing classical coding theory techniques for the design of DNA codes. Later, alphabets of size $4^{k}$ for $k \geq 1$ have been considered (e.g. [1, 4, 6-9, 13, 21? , 22]). It was also observed that having a cyclic structure in DNA codes has certain advantages in terms of complexities of algorithms [17, 21].

One of the challenging problems in the field is the reversibility problem [18]. This problem arises from the fact that the pairing of nucleotides in two different strands of a DNA sequence is done in opposite direction and reverse order. For example, let us consider the codeword (DNA string) GTTAGGCA which corresponds to a codeword $\left(a_{1}, a_{2}\right)$. The reverse of $\left(a_{1}, a_{2}\right)$ is $\left(a_{2}, a_{1}\right)$. However, the vector $\left(a_{2}, a_{1}\right)$ corresponds to $G G C A G T T A$ which is not the reverse of GTTAGGCA. The reverse of $G T T A G G C A$ is ACGGATTG.

Some authors solved this problem by considering skew cyclic codes. The reversibility problem for DNA 8-bases and DNA $2^{s+1} k$-bases is solved in [10] and [11] respectively by using skew cyclic codes over the finite rings $\mathbb{F}_{16}+u \mathbb{F}_{16}+v \mathbb{F}_{16}+u v \mathbb{F}_{16}$, where $u^{2}=u, v^{2}=v, u v=v u$ and $\mathbb{F}_{42 k}\left[u_{1}, \ldots, u_{s}\right] /\left\langle u_{1}^{2}-\right.$ $\left.u_{1}, \ldots, u_{s}^{2}-u_{s}\right\rangle$ where $k, s \geqslant 1, u_{i} u_{j}=u_{j} u_{i}$.

Motivated by the previous work [10, 11], we study the reversibility problem for DNA 4-bases using skew cyclic codes over the finite ring $R:=\mathbb{F}_{2}+u \mathbb{F}_{2}+v \mathbb{F}_{2}+w \mathbb{F}_{2}+u v \mathbb{F}_{2}+u w \mathbb{F}_{2}+v w \mathbb{F}_{2}+u v w \mathbb{F}_{2}$ of order 256 where $u^{2}=0, v^{2}=v, w^{2}=w, u v=v u, u w=w u, v w=w v$.

In [6], cyclic DNA codes over $R$ are studied. A map from $R$ to $R_{1}^{2}$ is given where $R_{1}=\mathbb{F}_{2}+u \mathbb{F}_{2}+$ $v \mathbb{F}_{2}+u v \mathbb{F}_{2}$ and $u^{2}=0, v^{2}=v, u v=v u$. Moreover, cyclic codes of arbitrary length over $R$ satisfying the reverse constraint and reverse complement constraint are studied and a one to one correspondence between the elements of the ring $R$ and $S_{D_{256}}$ is established where $S_{D_{256}}=\{A A A A, \ldots, G G G G\}$. The binary image of a cyclic code over $R$ is also determined.

In this paper, by defining a non-trivial automorphism on $R$, skew cyclic codes over $R$ are introduced. Thanks to these type of codes, reversible DNA codes are obtained and some examples are provided.

\section{Preliminaries}

In [6], the finite ring $R=\mathbb{F}_{2}+u \mathbb{F}_{2}+v \mathbb{F}_{2}+w \mathbb{F}_{2}+u v \mathbb{F}_{2}+u w \mathbb{F}_{2}+v w \mathbb{F}_{2}+u v w \mathbb{F}_{2}=\left\{a_{1}+u a_{2}+v a_{3}+\right.$ $\left.w a_{4}+u v a_{5}+u w a_{6}+v w a_{7}+u v w a_{8}: a_{i} \in \mathbb{F}_{2}, i=1,2, \ldots, 8\right\}$ with $u^{2}=0, v^{2}=v, w^{2}=w, u v=v u, u w=$ $w u, v w=w v$ is introduced. The ring $R$ is commutative with characteristic 2 and 256 elements. It can be viewed as

$$
\begin{aligned}
R & =\left(\mathbb{F}_{2}+u \mathbb{F}_{2}+v \mathbb{F}_{2}+u v \mathbb{F}_{2}\right)+w\left(\mathbb{F}_{2}+u \mathbb{F}_{2}+v \mathbb{F}_{2}+u v \mathbb{F}_{2}\right) \\
& =R_{1}+w R_{1}, \quad w^{2}=w
\end{aligned}
$$

where $R_{1}$ is the $\operatorname{ring} \mathbb{F}_{2}+u \mathbb{F}_{2}+v \mathbb{F}_{2}+u v \mathbb{F}_{2}, u^{2}=0, v^{2}=v, u v=v u$, introduced in [23]. By using the DNA alphabet $S_{D_{4}}=\{A, T, C, G\}$, the authors define a correspondence $\tau$ between the elements of the finite ring $R_{1}$ and DNA double pairs as in the following table, by means of a Gray map from $R_{1}$ to $\left(\mathbb{F}_{2}+u \mathbb{F}_{2}\right)^{2}$ with $u^{2}=0$. 


$\begin{array}{ll}\text { Ring elements } \alpha & \text { DNA double pairs } \tau(\alpha) \\ 0 & A A \\ 1 & G G \\ u & T T \\ v & A G \\ u v & A T \\ 1+u & C C \\ 1+v & G A \\ u+v & T C \\ u+u v & T A \\ v+u v & A C \\ 1+u v & G C \\ 1+u+u v & C G \\ 1+u+v & C T \\ 1+v+u v & G T \\ u+v+u v & T G \\ 1+u+v+u v & C A\end{array}$

We define a Gray map as follows

$$
\begin{array}{rll}
\phi & : \quad R \longrightarrow R_{1}^{2} \\
x+y w & \longmapsto & (x, x+y)
\end{array}
$$

where $x, y \in R_{1}$.

By using the matching and the Gray map above, we get a matching $\Psi$ between the elements of $R$ and a set of DNA 4-bases $S_{D_{256}}=\{A A A A, T T T T, \ldots\}$ as follows.

$$
\begin{aligned}
\Psi & : R \longrightarrow S_{D_{256}} \\
x+w y & \longmapsto \Psi(x+w y)=\gamma(x, x+y)=(\tau(x), \tau(x+y))
\end{aligned}
$$

where

$$
\begin{aligned}
\gamma & : \quad R_{1}^{2} \longrightarrow S_{D_{256}} \\
(s, t) & \longmapsto(\tau(s), \tau(t))
\end{aligned}
$$

for $s, t \in R_{1}$. That is, $\Psi=\gamma \circ \phi$.

\section{Skew cyclic codes over $R$}

Definition 3.1. Let $B$ be a finite ring and $\theta$ be a non-trivial automorphism on $B$. A subset $C$ of $B^{n}$ is called a skew cyclic code of length $n$ if $C$ satisfies the following conditions,

1. $C$ is a submodule of $B^{n}$

2. If $c=\left(c_{0}, c_{1}, \ldots, c_{n-1}\right) \in C$, then $\sigma_{\theta}(c)=\left(\theta\left(c_{n-1}\right), \theta\left(c_{0}\right), \ldots, \theta\left(c_{n-2}\right)\right) \in C$, where $\sigma_{\theta}$ is the skew cyclic shift operator.

By defining a non-trivial automorphism $\theta$ on $R$ as follows, we can define skew cyclic codes over $R$. Let

$$
\begin{aligned}
\theta & : R \longrightarrow R \\
x+y w & \longmapsto \theta(x+y w)=\theta^{\prime}(x+y)+w \theta^{\prime}(y)
\end{aligned}
$$


where $x, y \in R_{1}$ and $\theta^{\prime}$ is a non-trivial authomorphism on $R_{1}$ defined by

$$
\begin{aligned}
\theta^{\prime} & : R_{1} \longrightarrow R_{1} \\
a+b u+v(c+d u) & \longmapsto(a+c+(b+d) u)+v(c+d u)
\end{aligned}
$$

The order of each $\theta$ and $\theta^{\prime}$ is 2 .

The set of polynomials $R[x, \theta]=\left\{a_{0}+a_{1} x+\ldots+a_{n-1} x^{n-1}: a_{i} \in R, n \in \mathbb{N}\right\}$ is the skew polynomial ring over $R$ with the usual addition of polynomials and the non-commutative multiplication given by

$$
\left(a x^{i}\right)\left(b x^{j}\right)=a \theta^{i}(b) x^{i+j} .
$$

In polynomial representation, a skew cyclic code of length $n$ over $R$ is defined as a left ideal of the quotient ring $R_{\theta, n}=R[x, \theta] /\left\langle x^{n}-1\right\rangle$, if the order of $\theta$ divides $n$, that is, if $n$ is even. If the order of $\theta$ does not divide $n$, a skew cyclic code of length $n$ over $R$ is defined as a left $R[x, \theta]$-submodule of $R_{\theta, n}$, since the set $R_{\theta, n}=R[x, \theta] /\left\langle x^{n}-1\right\rangle=\left\{f(x)+\left\langle x^{n}-1\right\rangle: f(x) \in R[x, \theta]\right\}$ is a left $R[x, \theta]$-module with the multiplication from left defined by

$$
r(x)\left(f(x)+\left\langle x^{n}-1\right\rangle\right)=r(x) f(x)+\left\langle x^{n}-1\right\rangle
$$

for any $r(x) \in R[x, \theta]$.

In either case, the following holds.

Theorem 3.2. Let $C$ be a skew cyclic code over $R$ and let $f(x)$ be a polynomial in $C$ of minimal degree. If the leading coefficient of $f(x)$ is a unit in $R$, then $C=\langle f(x)\rangle$, where $f(x)$ is a right divisor of $x^{n}-1$.

Proof. It can be proven similarly to the proof of Theorem 4 in [20].

\section{Reversible DNA codes from skew cyclic codes over $R$}

Definition 4.1. For $x=\left(x_{0}, x_{1}, \ldots, x_{n-1}\right) \in R^{n}$, the vector $\left(x_{n-1}, x_{n-2}, \ldots, x_{1}, x_{0}\right)$ is called the reverse of $x$ and is denoted by $x^{r}$. A linear code $C$ of length $n$ over $R$ is said to be reversible if $x^{r} \in C$ for every $x \in C$.

Each element $\alpha$ of $R_{1}$ and $\theta^{\prime}(\alpha)$ are mapped to DNA pairs, which are reverses of each other. For example, $\tau(v)=A G$, while $\tau\left(\theta^{\prime}(v)\right)=G A$.

This map can be extended to a map $\gamma$ from $R_{1}^{2}$ to 4 -mers as follows,

$$
\gamma(a, b)=(\tau(a), \tau(b))
$$

where $a, b \in R_{1}$.

By means of the map $\Psi=\gamma \circ \phi$, we can find a relationship between skew cyclic codes over $R$ and DNA codes. We note that $\Psi(r)$ and $\Psi(\theta(r))$ are DNA reverses of each other. Indeed, for $r=x+y w \in R$, we have

$$
\begin{aligned}
\Psi(r) & =\gamma(\phi(x+y w))=\gamma(x, x+y) \\
& =(\tau(x), \tau(x+y))
\end{aligned}
$$

On the other hand,

$$
\begin{aligned}
\Psi(\theta(r)) & =\Psi\left(\theta^{\prime}(x+y)+w \theta^{\prime}(y)\right) \\
& =\gamma\left(\phi\left(\theta^{\prime}(x+y)+w \theta^{\prime}(y)\right)\right) \\
& =\gamma\left(\theta^{\prime}(x+y), \theta^{\prime}(x)\right) \\
& =\left(\tau\left(\theta^{\prime}(x+y)\right), \tau\left(\theta^{\prime}(x)\right)\right)
\end{aligned}
$$


This map can be extended as follows. For any $r=\left(r_{0}, \ldots, r_{n-1}\right) \in R^{n}$,

$$
\left(\Psi\left(r_{0}\right), \Psi\left(r_{1}\right), \ldots, \Psi\left(r_{n-1}\right)\right)^{r}=\left(\Psi\left(\theta\left(r_{n-1}\right)\right), \ldots, \Psi\left(\theta\left(r_{1}\right)\right), \Psi\left(\theta\left(r_{0}\right)\right)\right)
$$

Example 4.2. If $r=u+u v+w(1+u v) \in R$, then we get

$$
\begin{aligned}
\Psi(r) & =\gamma(\phi(r))=\gamma(u+u v, 1+u) \\
& =(\tau(u+u v), \tau(1+u))=(T A, C C)
\end{aligned}
$$

On the other hand,

$$
\begin{aligned}
\Psi(\theta(r)) & =\Psi\left(\theta^{\prime}(1+u)+w \theta^{\prime}(1+u v)\right) \\
& =\gamma \circ \phi\left(\theta^{\prime}(1+u)+w \theta^{\prime}(1+u v)\right) \\
& =\gamma\left(\theta^{\prime}(1+u), \theta^{\prime}(u+u v)\right) \\
& =\left(\tau\left(\theta^{\prime}(1+u)\right), \tau\left(\theta^{\prime}(u+u v)\right)\right) \\
& =(C C, A T)
\end{aligned}
$$

Definition 4.3. Let $C$ be a code of length $n$ over $R$. If $\Psi(c)^{r} \in \Psi(C)$ for all $c \in C$, then $C$ or equivalently $\Psi(C)$ is called a reversible DNA code.

Definition 4.4. Let $g(x)=a_{0}+a_{1} x+a_{2} x^{2}+\ldots+a_{s} x^{s}$ be a polynomial of degree s over $R$. $g(x)$ is called a palindromic polynomial if $a_{i}=a_{s-i}$ for all $i \in\{0,1, \ldots, s\} . g(x)$ is called a $\theta$-palindromic polynomial if $a_{i}=\theta\left(a_{s-i}\right)$ for all $i \in\{0,1, \ldots, s\}$.

As the order of $\theta$ is 2, a skew cyclic code of odd length $n$ over $R$ with respect to $\theta$ is an ordinary cyclic code. So we will take the length $n$ to be even.

The next two theorems show that palindromic and $\theta$-palindromic polynomials generate reversible DNA codes. They are analogous to Theorem 1 and Theorem 2 in [12] stated for codes over a field.

Theorem 4.5. Let $C=\langle f(x)\rangle$ be a skew cyclic code of length $n$ over $R$, where $f(x)$ is a right divisor of $x^{n}-1$ and $\operatorname{deg}(f(x))$ is odd. If $f(x)$ is a $\theta$-palindromic polynomial, then $\Psi(C)$ is a reversible DNA code.

Proof. Let $f(x)$ be a $\theta$-palindromic polynomial and $f(x)=a_{0}+a_{1} x+\ldots+a_{2 s-1} x^{2 s-1}$. So $a_{i}=$ $\theta\left(a_{2 s-1-i}\right)$, for all $i=0,1, \ldots, s-1$. Let $h(x)=h_{0}+h_{1} x+\cdots+h_{2 k-1} x^{2 k-1}$. Let $b_{l}$ be the coefficient of $x^{l}$ in $h(x) f(x)$, where $l=0,1, \ldots, n-1$. For any $t<n / 2$, the coefficient of $x^{t}$ in $h(x) f(x)$ is

$$
b_{t}=\sum_{j=0}^{t} h_{j} \theta^{j}\left(a_{t-j}\right)
$$

and the coefficient of $x^{n-t}$ is $b_{n-t}=\sum_{j=0}^{t} h_{2 k-1-j} \theta^{2 k-1-j}\left(a_{2 s-1-(t-j)}\right)$.

The polynomial $h(x) f(x)=\sum_{d=0}^{2 k-1} h_{d} x^{d} f(x)$ corresponds to a vector $b=\left(b_{0}, b_{1}, \ldots, b_{n-1}\right) \in C$.

The vector $\Psi(b)^{r}=\left(\left(\Psi\left(b_{0}\right), \ldots, \Psi\left(b_{n-1}\right)\right)\right)^{r}$ is equal to the vector $\Psi(z)$, where the vector $z$ corresponds to the polynomial $\sum_{d=0}^{2 k-1} \theta\left(h_{d}\right) x^{2 k-1-d} f(x)$.

So, $\Psi(C)$ is a reversible DNA code.

Theorem 4.6. Let $C=\langle f(x)\rangle$ be a skew cyclic code of length $n$ over $R$, where $f(x)$ is a right divisor of $x^{n}-1$ and $\operatorname{deg}(f(x))$ is even. If $f(x)$ is a palindromic polynomial, then $\Psi(C)$ is a reversible DNA code. 
Proof. Let $f(x)$ be a palindromic polynomial with even degree so that $f(x)=a_{0}+a_{1} x+\ldots+a_{2 s} x^{2 s}$ and $a_{i}=a_{2 s-i}$, for all $i=0,1, \ldots, s$. Let $h(x)=h_{0}+h_{1} x+\ldots+h_{2 k} x^{2 k}$. Let $b_{l}$ be the coefficient of $x^{l}$ in $h(x) f(x)$, where $l=0,1, . ., n-1$. For any $t<n / 2$, the coefficient of $x^{t}$ in $h(x) f(x)$ is

$$
b_{t}=\sum_{j=0}^{t} h_{j} \theta^{j}\left(a_{t-j}\right)
$$

and the coefficient of $x^{n-t}$ is $b_{n-t}=\sum_{j=0}^{t} h_{(2 k)-j} \theta^{(2 k)-j}\left(a_{2 s-(t-j)}\right)$.

The polynomial $h(x) f(x)=\sum_{d=0}^{2 k} h_{d} x^{d} f(x)$ corresponds to a vector $b=\left(b_{0}, b_{1}, \ldots, b_{n-1}\right) \in C$.

The vector $\Psi(b)^{r}=\left(\left(\Psi\left(b_{0}\right), \ldots, \Psi\left(b_{n-1}\right)\right)\right)^{r}$ is equal to the vector $\Psi(z)$, where the vector $z$ corresponds to the polynomial $\sum_{d=0}^{2 k} \theta\left(h_{d}\right) x^{2 k-d} f(x)$. So, $\Psi(C)$ is a reversible DNA code.

The next two theorems show that palindromic and $\theta$-palindromic polynomials come in pairs. They are analogous to Theorem 4 and Theorem 3 in [12] stated for skew polynomials over a field.

Theorem 4.7. Let $x^{n}-1=h(x) f(x) \in R[x, \theta]$, where the degree of $f(x)$ is odd. If $f(x)$ is a $\theta$-palindromic polynomial, then $h(x)$ is a palindromic polynomial.

Proof. Let $f(x)=a_{0}+a_{1} x+\ldots+a_{2 s-1} x^{2 s-1}$. As the length $n$ is even, then $h(x)=h_{0}+h_{1} x+\ldots+$ $h_{2 k-1} x^{2 k-1}$. Since $f(x)$ is a $\theta$-palindromic polynomial, then $a_{i}=\theta\left(a_{2 s-1-i}\right)$ for all $i=0,1, \ldots, s-1$. Let $b_{l}$ be the coefficient of $x^{l}$ in $h(x) f(x)$, where $l=0,1, . ., n-1$. For any $t<n / 2$, the coefficient of $x^{t}$ in $h(x) f(x)$ is

$$
b_{t}=\sum_{j=0}^{t} h_{j} \theta^{j}\left(a_{t-j}\right)
$$

and the coefficient of $x^{n-t}$ is $b_{n-t}=\sum_{j=0}^{t} h_{2 k-1-j} \theta^{2 k-1-j}\left(a_{2 s-1-(t-j)}\right)$. By using the fact that $b_{0}=$ $b_{n}=0$ and $b_{i}=0$ for all $i=1,2, \ldots, n-1$, it can be shown that $h_{i}=h_{2 k-1-i}$ for all $i=0,1, . ., k-1$ as in the proof of Theorem 4 in [12], by induction.

A polynomial that is in the center $Z(R[x, \theta])$ of $R[x, \theta]$ is called a central polynomial. A central polynomial commutes with every element of $R[x, \theta]$. As in the field case, we can prove that $x^{n}-1$ is central when $n$ is even.

Proposition 4.8. The polynomial $x^{n}-1$ is central if and only if $n$ is even.

Proof. Let $n$ be even. Let $s(x)=a_{0}+a_{1} x+\ldots+a_{m} x^{m} \in R[x, \theta]$. Since $n$ is even, $\theta^{n}(a)=a$ for any $a \in R$. So $\left(x^{n}-1\right) s(x)=x^{n} a_{0}+x^{n} a_{1} x+\ldots+x^{n} a_{m} x^{m}-s(x)=x^{n} a_{0}+\theta^{n}\left(a_{1}\right) x^{n} x+\ldots+\theta^{n}\left(a_{m}\right) x^{n} x^{m}-s(x)=$ $\left(a_{0}+a_{1} x+\ldots+a_{m} x^{m}\right) x^{n}-s(x)=s(x)\left(x^{n}-1\right)$. Hence $\left(x^{n}-1\right) \in Z(R[x, \theta])$. Conversely, assume $\left(x^{n}-1\right) \in Z(R[x, \theta])$. Then $\left(x^{n}-1\right)$ commutes with every element in $R[x, \theta]$. In particular, we have $\left(x^{n}-1\right) a_{m} x^{m}=a_{m} x^{m}\left(x^{n}-1\right)$ for any $m$ and any $a_{m} \in R$. As $\left(x^{n}-1\right) a_{m} x^{m}=\theta^{n}\left(a_{m}\right) x^{n+m}-a_{m} x^{m}$ and $a_{m} x^{m}\left(x^{n}-1\right)=\left(a_{m}\right) x^{n+m}-a_{m} x^{m}$, we have $\theta^{n}\left(a_{m}\right)=a_{m}$. This implies that $n$ is even.

Lemma 4.9. Let $x^{n}-1=h f \in Z(R[x, \theta])$. Then $h f=f h$ in $R[x, \theta]$.

Proof. Since $h f$ is a central element, we have $(h f) h=h(h f)$. So $h(f h-h f)=0$. Since the leading coefficient of $h$ is a unit, $h$ is not a zero divisor. Hence, $f h=h f$ in $R[x, \theta]$.

Theorem 4.10. Let $x^{n}-1=h(x) f(x) \in R[x, \theta]$, where the degree of $f(x)$ is even. If $h(x)$ is a palindromic polynomial then so is $f(x)$. 
Proof. This can be proven similarly to Theorem 3 in [12].

Since $n$ is even, $x^{n}-1=h f \in Z(R[x, \theta])$. So we get that any right divisor of $x^{n}-1$ is also a left divisor of $x^{n}-1$.

Corollary 4.11. Let $x^{n}-1=h(x) f(x) \in R[x, \theta]$, where the degree of $f(x)$ is even. If $f(x)$ is a palindromic polynomial, then $h(x)$ is a palindromic polynomial as well.

Finally, we give a few examples of palindromic and theta-palindromic polynomials over $R$. Hence, these polynomials generate reversible DNA codes. We found these polynomials using Magma software $[15]$.

Example 4.12. There are at least 576 different factorizations of $x^{4}-1$ in the form $x^{4}-1=f(x) h(x)$ where $\operatorname{deg}(f(x))=\operatorname{deg}(h(x))=2$, in the skew polynomial ring over $R$. Of these, 64 of the factorizations involve palindromic polynomials. One of these 64 pairs is $f(x)=h(x)=x^{2}+u x+1$.

Example 4.13. There are at least 990 different factorizations of $x^{8}-1$ in the form $x^{8}-1=h(x) f(x)$ over $R$ where $\operatorname{deg}(h(x))=3$ and $\operatorname{deg}(f(x))=5$. Of these, 1 factorization involves theta-palindromic polynomials, namely

$h(x)=x^{3}+(u w+(u v+(u+1))) x^{2}+(u w+(u v+(u+1))) x+1$ and

$f(x)=x^{5}+(u w+(u v+(u+1))) x^{4}+(u w+(u v+u)) x^{3}+(u w+(u v+u)) x^{2}+(u w+(u v+(u+1))) x+1$.

Example 4.14. The polynomial $f(x)=x^{4}+(v+1) x^{3}+(u v w+u) x^{2}+(v+1) x+1$ divides $x^{16}-1$ in the skew polynomial ring over $R$ and it is palindromic. Hence it generates a reversible DNA code. Additionally, $h(x)=\frac{x^{16}-1}{f(x)}$ (division in the skew polynomial ring over $R$ ) is also palindromic.

Example 4.15. There are at least 1600 different factorizations of $x^{12}-1$ in the form $x^{12}-1=f(x) h(x)$ in the skew polynomial ring over $R$ where $\operatorname{deg}(f(x))=2$ and $\operatorname{deg}(h(x))=10$. Of these, 144 of factorizations involve palindromic polynomials, one of which is $f(x)=x^{2}+(u v+u) x+1$.

\section{Conclusion}

We have shown that skew cyclic codes over the ring $R=\mathbb{F}_{2}+u \mathbb{F}_{2}+v \mathbb{F}_{2}+w \mathbb{F}_{2}+u v \mathbb{F}_{2}+u w \mathbb{F}_{2}+$ $v w \mathbb{F}_{2}+u v w \mathbb{F}_{2}$, where $u^{2}=0, v^{2}=v, w^{2}=w, u v=v u, u w=w u, v w=w v$, can be used to construct the reversible DNA codes. We have also provided several specific examples of such codes.

\section{References}

[1] T. Abualrub, A. Ghrayeb, X. N. Zeng, Construction of cyclic codes over GF(4) for DNA computing, J. Frankl. Inst. 343(4-5) (2006) 448-457.

[2] L. Adleman, Molecular computation of the solutions to combinatorial problems, Science 266 (1994) 1021-1024.

[3] L. Adleman, P. W. K. Rothemund, S. Roweis, E. Winfree, On applying molecular computation to the data encryption standard, J. Comp. Biology 6(1) (1999) 53-63.

[4] N. Bennenni, K. Guenda, S. Mesnager, DNA cyclic codes over rings, Advances in Mathematics of Communications 11(1) (2017) 83-98.

[5] D. Boneh, C. Dunworth, R. Lipton, Breaking DES using molecular computer, Princeton CS TechReport, Number CS-TR-489-95 (1995).

[6] Y. Cengellenmis, A. Dertli, On the cyclic DNA codes over the finite ring, Acta Universitatis Apulensis 58 (2019) 1-11. 
[7] A. Dertli, Y. Cengellenmis, On cyclic DNA codes over the rings $Z_{4}+w Z_{4}$ and $Z_{4}+w Z_{4}+v Z_{4}+w v Z_{4}$, Biomath 6(2) (2017) 1712167.

[8] P. Gaborit, H. King, Linear constructions for DNA codes, Theor. Comput. Sci. 334(1âĂŞ3) (2005) 99-113.

[9] K. Guenda, T. A. Gulliver, Construction of cyclic codes over $\mathbb{F}_{2}+u \mathbb{F}_{2}$ for DNA computing, AAECC 24 (2013) 445-459.

[10] F. Gursoy, E. S. Oztas, I. Siap, Reversible DNA codes over $\mathbb{F}_{16}+u \mathbb{F}_{16}+v \mathbb{F}_{16}+u v \mathbb{F}_{16}, 11(2) 2017$ 307-312.

[11] F. Gursoy, E. S. Oztas, B. Yildiz, Reversible DNA codes over a family of non-chain ring, arXiv:1711.02385.

[12] F. Gursoy, E. S. Oztas, I. Siap, Reversible DNA codes using skew polynomial rings, Applicable Algebra in Engineering, Communication and Computing 28 (2017) 311-320.

[13] J. Liang, L. Wang, On cyclic DNA codes over $\mathbb{F}_{2}+u \mathbb{F}_{2}$, J.Appl Math Comput. 52 (2016) 81-91.

[14] D. Limbachiya, B. Rao, G. K. Manish, The Art of DNA Strings: Sixteen Years of DNA Coding Theory, arXiv:1607.00266.

[15] Magma computer algebra system, online, http://magma.maths.usyd.edu.au/

[16] M. Mansuripur, P. K. Khulbe, S. M. Kuebler, J. W. Perry, M. S. Giridhar, N. Peyghambarian, Information storage and retrieval using macromolecules as storage media, in Optical Data Storage, OSA Technical Digest Series (Optical Society of America), paper TuC2 (2003).

[17] O. Milenkovic, N. Kashyap, On the design of codes for DNA computing, Lecture Notes in Computer Science 3969, Springer (2006) 100-119.

[18] E. S. Oztas, B. Yildiz and I. Siap, A novel approach for constructing reversible codes and applications to DNA codes over the ring $\mathbb{F}_{2}[u] /\left(u^{2 k}-1\right)$, Finite Fields and Their Applications 46 (2017) 217-234.

[19] S. Pattanayak, A. K. Singh, Construction of cyclic DNA codes over the Ring $Z_{4}[u] /<u^{2}-1>$ based on the deletion distance, arXiv:1603.04055.

[20] A. Sharma, B. Maheshanand, A class of skew-constacyclic codes over $Z_{4}+u Z_{4}$, International Journal of Information and Coding Theory 4(4) (2017) 289-303.

[21] I. Siap, T. Abualrub, A. Ghrayeb, Cyclic DNA codes over the ring $\mathbb{F}_{2}[u] /\left(u^{2}-1\right)$ based on the deletion distance, J. Frankl. Inst. 346 (2009) 731-740.

[22] B. Yildiz, I. Siap, Cyclic codes over $\mathbb{F}_{2}[u] /\left(u^{4}-1\right)$ and applications to DNA codes, Comput. Math. Appl. 63 (2012) 1169-1176.

[23] S. Zhu, X. Chen, Cyclic DNA codes over $\mathbb{F}_{2}+u \mathbb{F}_{2}+v \mathbb{F}_{2}+u v \mathbb{F}_{2}$ and their applications, J. Appl.Math Comput. 55 (2017) 479-493. 